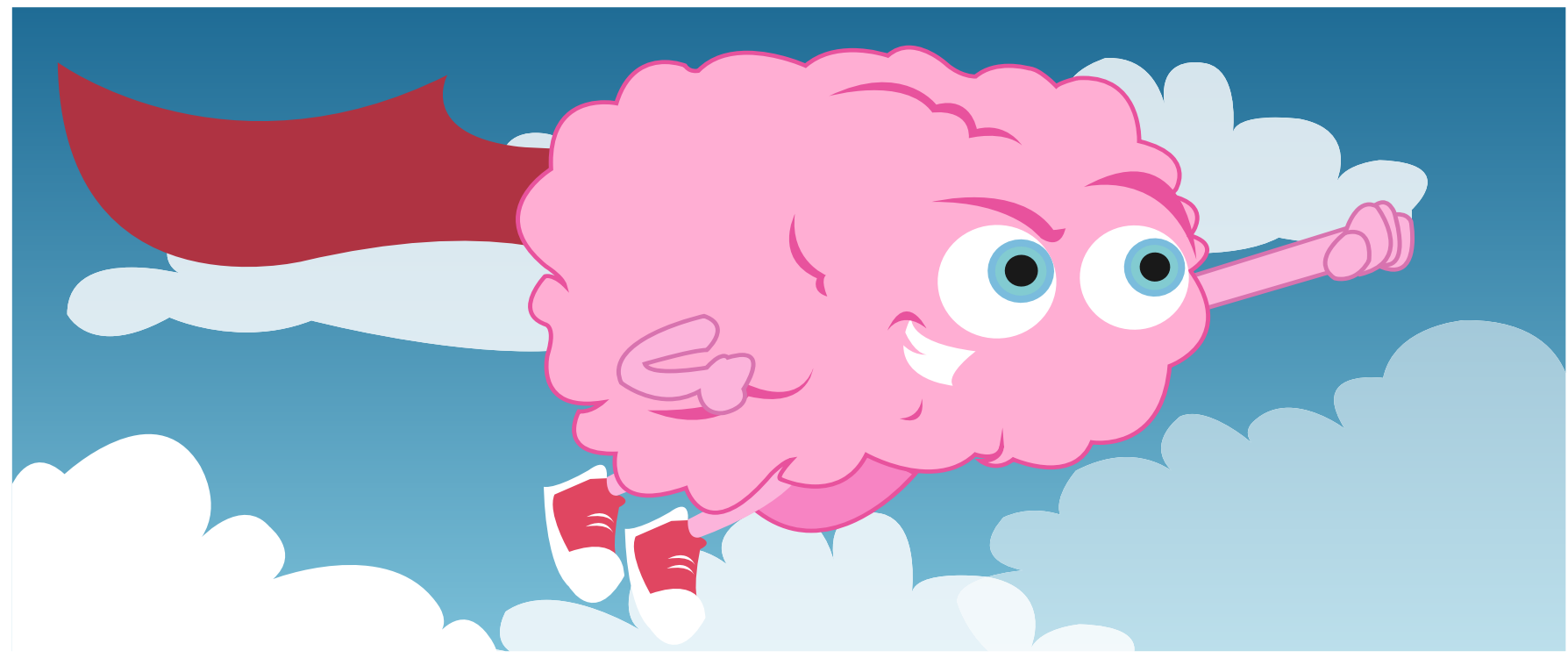

\title{
HAVE NO FEAR, THE BRAIN IS HERE! HOW YOUR BRAIN RESPONDS TO STRESS
}

\section{Kylie Garber Bezdek ${ }^{*}$ and Eva H. Telzer ${ }^{2}$}

${ }^{1}$ Frank Porter Graham Child Development Institute, Psychology and Neuroscience, University of North Carolina at Chapel Hill, Chapel Hill, NC, United States, ²Developmental Social Neuroscience Laboratory, Psychology and Neuroscience, University of North Carolina at Chapel Hill, Chapel Hill, NC, United States

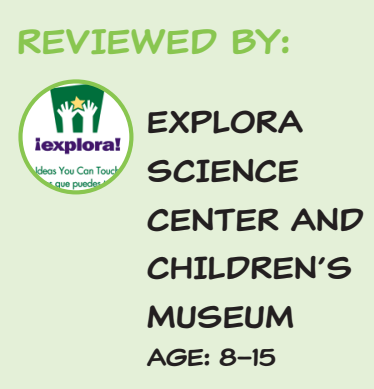

If you encountered a bear in the woods what would you do? In this article, we will talk about what stress is and how our brains and bodies react to it. There are many cool things that happen inside of the human body when we are faced with a scary situation. We will focus on the brain regions that are responsible for our reactions to stress. We will learn how they help our bodies to calm down when faced with something scary. The main parts of the brain that are responsible for our reactions to stress include the hypothalamic-pituitary-adrenal axis, the amygdala, and the prefrontal cortex. This article will also cover how the brain gets help from outside sources and how humans adapt to stress when it becomes a normal part of life. Let us read all about the superheroes that help us to overcome even the scariest situations!

\section{WHAT IS THE STRESS RESPONSE SYSTEM?}

Imagine how you would feel if you encountered a bear in the woods. Your heart might start racing and you might start breathing heavily. You might freeze in 


\section{STRESS}

The mental and physical state humans feel when they experience something difficult or threatening.

STRESS

RESPONSE

SYSTEM

The name for the parts of the brain, organs, and hormones that work together to combat stress.

HYPOTHALAMUSPITUITARY ADRENAL (HPA) AXIS

The messenger system that begins in the brain. It signals the organs to react to stress by going into survival mode. It includes the hypothalamus, the pituitary, and the adrenal gland.

\section{CORTISOL}

The stress hormone, or messenger, that is released by the HPA axis to tell other organs in the body to deal with a stressor.

\section{AMYGDALA}

The brain structure that actually detects stress and tells the HPA axis to respond.

place, unable to move out of fear. You might feel the urge to run away. These are all symptoms of stress. Stress is the mental and physical state humans feel when they experience something difficult or threatening. Stress can come from many different sources. Normal stressors are things that make you feel nervous or scared for a short time, like talking in front of a large group of people. Bigger, long-lasting stressors make you feel sad or scared for a long time. The death of a close family member is one example. The good news is that your brain is a superhero! Every day it keeps you safe from too much stress.

The brain gets help from other organs to calm you down when you face scary or sad stressful situations. The stress-response system is the name of the team of superheroes in your body that is led by the brain to combat stress. The stress-response system takes action by speeding up your heart beat to increase blood flow, speeding up your breathing to take in more oxygen, and slowing your digestion to store away fat and sugar for energy. In this article, we will talk about how the brain and body react to stress and how the brain regulates these reactions. We will also talk about the outside help the brain uses to regulate stress because even superheroes need a little help sometimes! Finally, we will learn how humans adapt to stress when it is long lasting.

\section{SUPERHEROES OF THE STRESS RESPONSE SYSTEM}

When the brain detects stress in the environment, the stress-response system goes into action. This begins with the hypothalamus-pituitary-adrenal (HPA) axis (Figure 1). Those are some very long words, so scientists just call it the HPA axis. When the brain detects stress, it first sends a message to a part of the brain called the hypothalamus. The job of the hypothalamus is to wake up the pituitary gland. Although the pituitary is only about the size of a small pea, it has a mighty job. The pituitary releases hormones, which are the messengers in the stress-response system. These hormones travel out of the brain to the adrenal glands. The adrenal glands sit on top of the kidneys. The adrenal glands release cortisol into the body.

Cortisol is known as the stress hormone. Cortisol is a messenger that sets other organs in the body into action. It is like the superpower of the stress response system. Cortisol helps the brain to think clearly, sends energy to important muscles, and increases heart rate and breathing. You can imagine that all of these bodily functions would be important if you were face-to-face with a bear: you would need to think about how to escape, use your muscles to run away, and have a fast heartbeat to pump lots of blood to the muscles and fast breathing to take in more oxygen [1].

Another important brain structure involved in the stress response system is called the amygdala. This funny-sounding brain structure is the size 


\section{FIGURE 1}

The hypothalamuspituitary-adrenal (HPA) axis acts to release cortisol into the blood stream. Cortisol calls the body into action to combat stress. Cortisol also regulates the HPA axis. When high amounts of cortisol interact with the hypothalamus, the HPA axis will slow down its activity. The amygdala detects stress in the environment, while the prefrontal cortex regulates our reactions to stress.

\section{PREFRONTAL \\ CORTEX}

The control center of the brain that controls thoughts and actions. Its main job is to control the emotional responses to stress by regulating the amygdala.

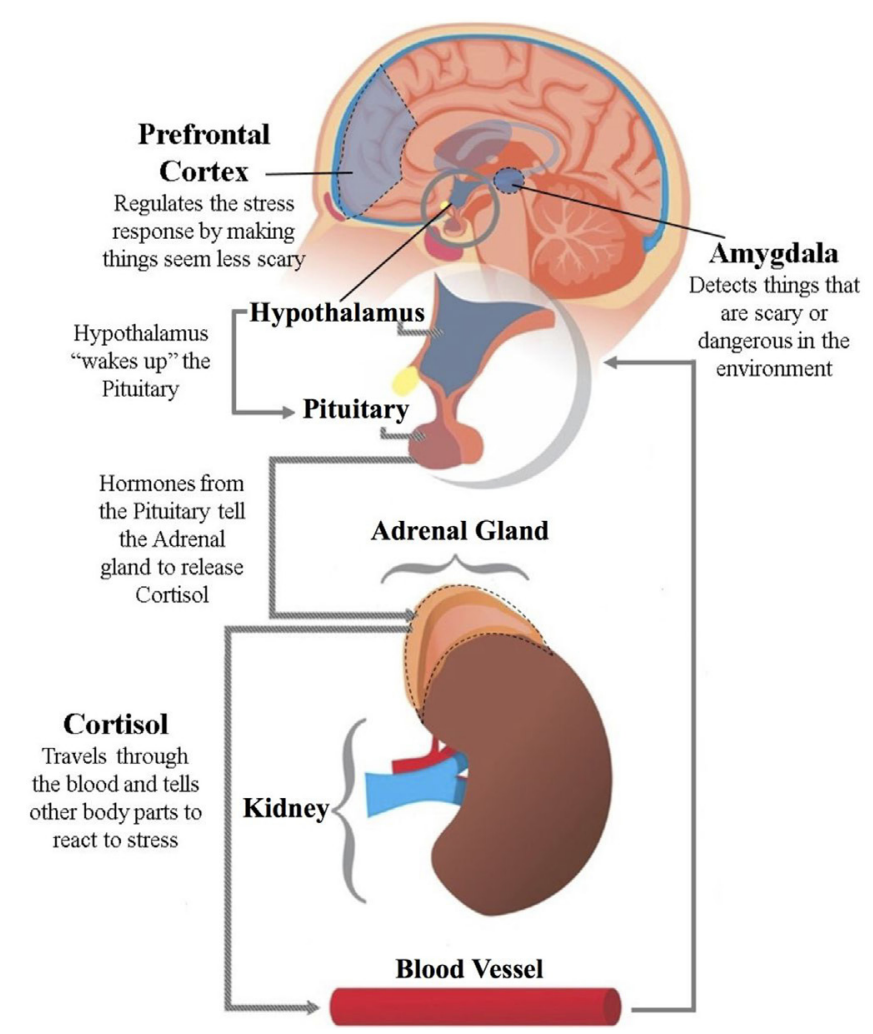

FIGURE 1

of a small kidney bean. It is located in the middle of the brain (Figure 1). The amygdala is the brain structure that actually detects stress and tells the HPA axis to respond. It can detect both emotional and biological stressors. An emotional stressor is something in the environment that may cause you to feel scared, sad, or frustrated, like the bear. A biological stressor is internal stress felt by the body, because of an injury or illness [1]. These functions of the amygdala are extremely important for survival. Just think-if you could not detect things that are harmful or stressful, you would not survive!

The amygdala shares a special connection with another part of the brain called the prefrontal cortex. The prefrontal cortex is a big region in the front of the brain (Figure 1). It can be called the control center of our brains because it helps to control our thoughts and actions. The main job of the prefrontal cortex is to control our emotional responses to stress so that we do not get too stressed out. This is why the amygdala and the prefrontal cortex share a special connection [2]. The amygdala quickly signals a threat or stress in the environment, and the prefrontal cortex helps the amygdala to see stressful events as a little less scary or frustrating. It is important to be able to use the brain to help slow the production of cortisol in the HPA axis. This process helps us calm down during a normal stressor by perceiving the situation as non-life threatening. In the bear example, which is a real danger, this process would help us to calm down after the bear runs away. 


\section{EVEN SUPERHEROS SOMETIMES NEED HELP}

Even though our bodies have these super stress-response systems, humans are best at dealing with stress when they have a little help. This help is called social support, which refers to the ways that other people can help us feel safe, loved, and cared for [1]. Your friends and family may provide social support by hugging you when you are sad or scared, hanging out with you when you feel lonely, or celebrating with you when you are excited. We especially need social support when we are very young. Remember earlier when we mentioned that the amygdala shares a special connection with the prefrontal cortex? This connection does not mature until you are a teenager; therefore, infants and children rely on their parents to help them calm down.

Scientists have studied how the brain responds to stress using a special technique called functional magnetic resonance imaging, or fMRI for short. fMRI is like a big camera that takes pictures of our insides using magnets. fMRI can help scientists detect which areas of the brain are active during certain tasks. Scientists did an experiment to find out how moms help their children deal with stress. Children (ages 4-10) and teenagers (ages 11-17) viewed emotional faces on a computer screen. Some of the faces showed negative emotions, like sadness or fear. Because seeing these negative emotional faces could be stressful, the amygdalas of the children and teenagers became active when these faces were viewed [2]. Children who had their mothers next to them as they viewed the faces showed lower amygdala activity (Figure 2). These children also had more mature connections between the amygdala and prefrontal cortex when their mothers were nearby! This means that the children' prefrontal cortex was activating more and their amygdala was activating less, helping the children feel less stressed. When people, like the moms in this experiment, provide social support that helps regulate the stress response, it is called

FIGURE 2

This study [2] compared the brains of children and teenagers while they viewed emotional faces. When looking at negative emotional faces (sad, angry, etc.) you can see that the children's amygdala activity was decreased when their mothers were present.

This tells us that the moms were buffering stress-response systems in the children by providing social support. The teenagers' amygdala activity increased when they viewed the emotional faces even though their mothers were present.

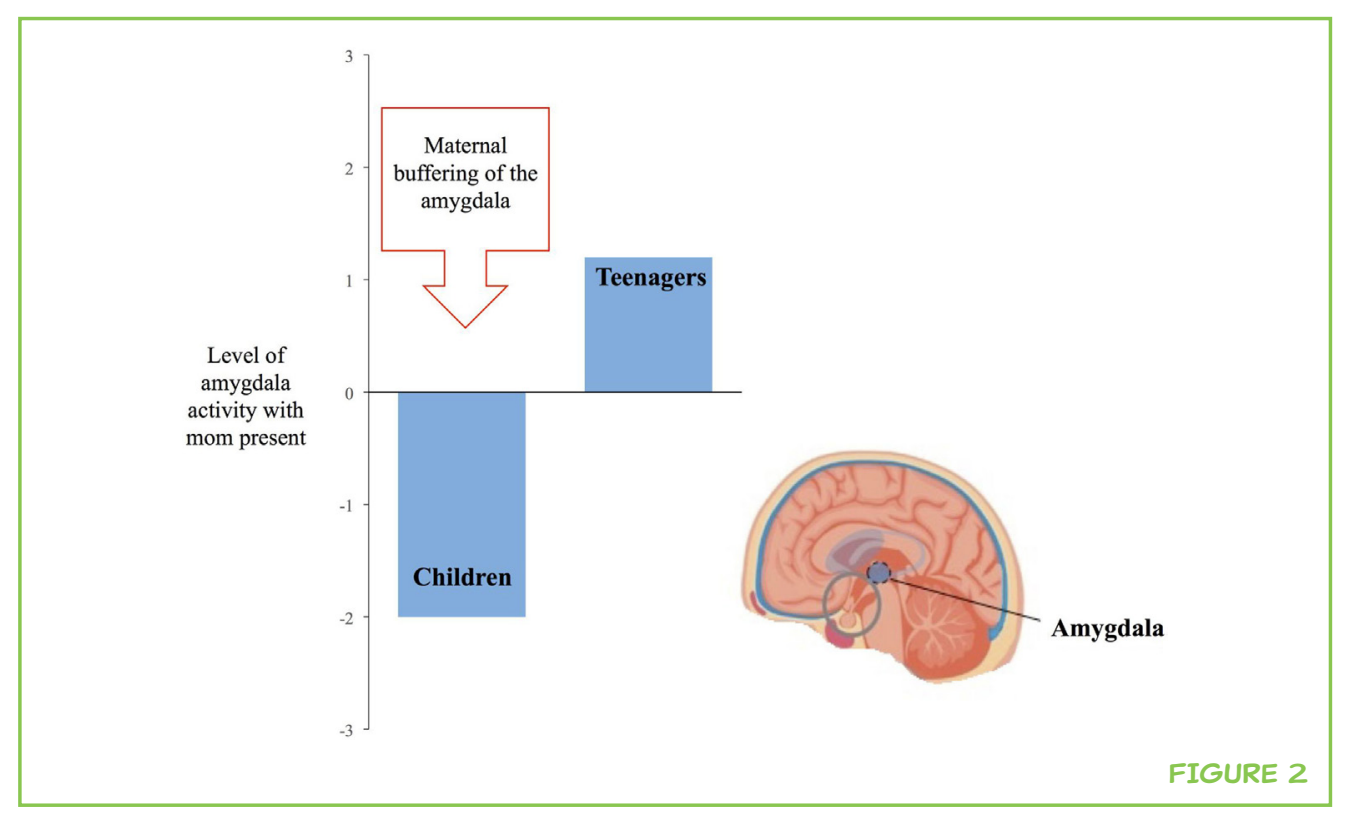


SOCIAL

BUFFERING

The process that happens when social support helps the brain regulate the stress response. Maternal buffering is social buffering that comes specifically from our mothers or other close caregivers

social buffering. Buffering means to protect or shield. In the experiment, we just talked about, the children's moms were buffering, or protecting the amygdala from too much activity. Social buffering that comes from mothers is called maternal buffering. Research has shown that moms and other caregivers (like dads and babysitters) help to lower the cortisol levels in babies and kids who have experienced a stressful situation [1].

The results of the study are even more amazing because the scientists did not observe maternal buffering when teenagers did the same task with their mothers next to them. Does this mean that teenagers no longer need their moms? Evidence from another study show that teens still need their moms, but in a different way. In this experiment, the scientists had teenagers play a risky driving video game alone and when their moms were present. In the game, teenagers approached a yellow light that was about to turn red. They had to decide if they should drive through the light and risk getting into a car accident. They found that teens made more safe choices when their moms were present, and just like the study above, the mom's presence helped the prefrontal cortex come into action [3]. This study demonstrates that teens also need the support of their moms to make good choices!

\section{WHAT HAPPENS WHEN STRESS LASTS A LONG TIME?}

Stress comes in many different forms. The example we have used throughout this article is encountering a bear. Seeing a bear is typically a short-term stressor, because you would probably get out of that stressful situation quickly. Feeling stressed is normal and good for detecting danger in the environment. But for some people, stress becomes a normal part of life. Imagine if you were bullied at school. Going to school every day might become scary or stressful. Short-term stress causes short bursts of a lot of cortisol. If the HPA axis is activated continuously, as with long-term stress, the stress-response system will change to try and deal with long-term stress [1]. The stress-response system changes by making less cortisol since there is so much in the body. This causes an imbalance of cortisol and poor functioning of the stress-response system.

One situation that might cause long-term stress is very poor care early in life, such as living in an orphanage without parents. A study (see Figure 3) compared children who lived in orphanages to children who grew up with their parents. Similar to the last study we talked about, the scientists looked at the children's brains while they viewed emotional faces [4]. They also compared the children' brains to teenagers' brains while they viewed these faces. The scientists also focused on the connection between the prefrontal cortex and the amygdala to study how these children regulated emotional stress. What they found was that the orphaned children's brains looked more like the teenagers' brains than the non-orphaned children. So the orphaned children's brains were 


\section{FIGURE 3}

The brain A. shows the connection between the amygdala and prefrontal cortex. The graph shows how the amygdala and prefrontal cortex are connected while the children and teenagers viewed emotional faces. Bars in the negative direction $\mathbf{B}$. indicate that the amygdala becomes less active because the prefrontal cortex becomes more active. This is what happens during regulation of emotional stress. Bars in the positive direction

C. indicate that both the amygdala and the prefrontal cortex are becoming more active. This is what happens when the brain is not able to regulate stress. You can see that the bars for orphaned children and teenagers both go in the same direction (negative). The bars for the nonorphaned children and teenagers go in opposite directions [4]. This tells us that in the absence of good early care these areas of the brain are maturing faster.

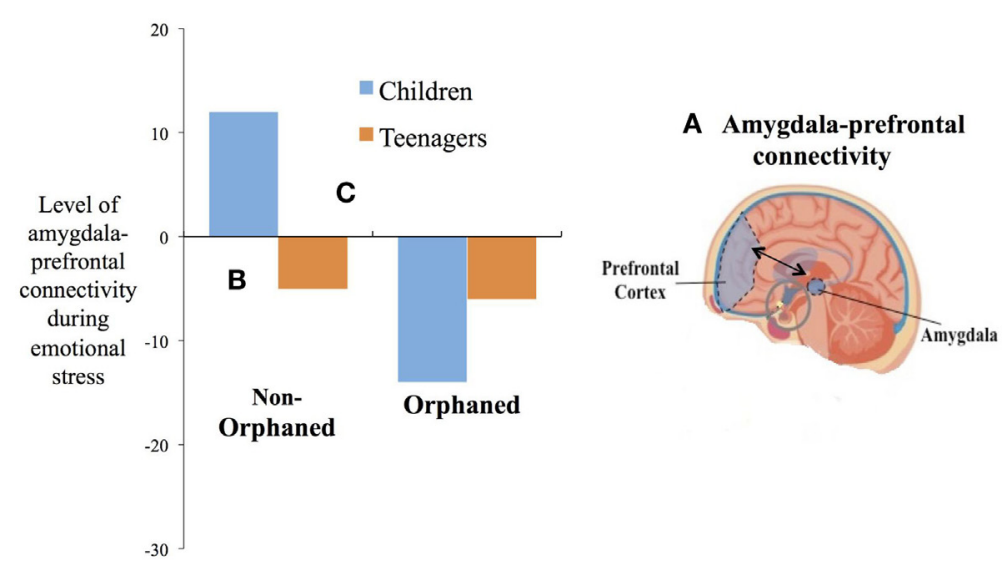

FIGURE 3

actually more mature! This means that their brains were able to regulate their emotional stress even without a parent nearby.

It might sound like a good thing to have a mature brain. But this is not always true for humans. Humans take a long time to mature. The normal pattern of human development allows humans to learn a lot about how to be an adult before actually becoming one. The children in this study lived in very crowded orphanages without much love and affection from adults. Without a close relationship with an adult as young children, the orphaned children's brains had to become mature much sooner. Becoming mature too soon is related to more anxiety, a mental state of worry and fear [4]. The good news is that children who are adopted eventually experience social buffering from stress when they feel loved and supported by their adopted family [1].

\section{CONCLUSION}

The brain fights stress every single day. Whether an enormous bear is chasing you or you see a small spider in your room, the brain and the body are ready and equipped to deal with the stress. Our brains are like superheroes-ready to save the day! With the help of the HPA axis, the amygdala, and the prefrontal cortex, we can calm ourselves down during stressful situations. No one can get through everything alone, not even superheroes. Our brains sometimes rely on the help of our friends and families to help buffer the response to stress. Parents are extremely helpful, especially when we are young. In the absence of a caregiver at a young age, a child's brain may become mature too fast, which may make a child feel anxiety. Anxiety makes things seem scarier than they actually are, which will make the child feel even more stressed! There are things you can do to handle short-term and long-term stress in your own life. Doing any activity that you enjoy releases chemicals in your brain that make you feel happy. Exercising and moving your body are great ways to reduce stress. Another way to handle stress is spending time with friends and family. 
Remember that having people in your life who provide social support can make you feel cared for, which will slow the release of cortisol. The best thing to do is tell a trusted adult if you are feeling stressed for a long period of time!

\section{REFERENCES}

1. Hostinar, C. E., Sullivan, R. M., and Gunnar, M. R. 2014. Psychobiological mechanisms underlying the social buffering of the hypothalamic-pituitaryadrenocortical axis: a review of animal models and human studies across development. Psychol. Bull. 140(1):256-82. doi:10.1037/a0032671

2. Gee, D. G., Gabard-Durnam, L., Telzer, E. H., Humphreys, K. L., Goff, B., Shapiro, M., et al. 2014. Maternal buffering of human amygdala-prefrontal circuitry during childhood but not during adolescence. Psychol. Sci. 25:2067-78. doi:10.1177/0956797614550878

3. Guassi Moreira, J. F., and Telzer, E. H. 2016. Mother still knows best: maternal influence uniquely modulated adolescent reward sensitivity during risk taking. Dev. Sci. 1-11. doi:10.1111/desc. 12484

4. Gee, D. G., Gabard-Durnam, L. J., Flannery, J., Goff, B., Humphreys, K. L., Telzer, E. H., et al. 2013. Early developmental emergence of human amygdalaprefrontal connectivity after maternal deprivation. Proc. Natl. Acad. Sci. U.S.A. 110(39):15638-43. doi:10.1073/pnas.1307893110

SUBMITTED: 17 August 2017; ACCEPTED: 07 December 2017; PUBLISHED ONLINE: 20 December 2017.

EDITED BY: Kathleen Haaland, University of New Mexico, United States

CITATION: Bezdek KG and Telzer EH (2017) Have No Fear, the Brain is Here! How Your Brain Responds to Stress. Front. Young Minds 5:71. doi:10.3389/frym.2017.00071

CONFLICT OF INTEREST STATEMENT: The authors declare that the research was conducted in the absence of any commercial or financial relationships that could be construed as a potential conflict of interest.

COPYRIGHT @ 2017 Bezdek and Telzer. This is an open-access article distributed under the terms of the Creative Commons Attribution License (CC BY). The use, distribution or reproduction in other forums is permitted, provided the original author(s) or licensor are credited and that the original publication in this journal is cited, in accordance with accepted academic practice. No use, distribution or reproduction is permitted which does not comply with these terms.

\section{REVIEWED BY}

EXPLORA SCIENCE CENTER AND CHILDREN'S MUSEUM, AGE: 8-15

The Explora Young Minds reviewers are a group of science enthusiasts working with museum educators and mentors from the University of New Mexico. We enjoy learning 
about the brain through the articles and hands-on activities and demonstrations. We also enjoy reading about new research, asking questions, and making suggestions to help the scientists make their work more understandable for everyone!

\section{AUTHORS}

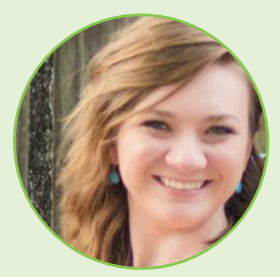

\section{KYLIE GARBER BEZDEK}

I am a graduate student at The University of North Carolina at Chapel Hill. I am interested in how early life experiences affect development throughout the lifespan. I am particularly interested in roles of early childcare and parenting in the development of children who grow up in poverty and rural areas of the United States. Currently, I am focusing on which characteristics of a child's early household environment have the biggest effect on kindergarten readiness skills (e.g., early math, language, and social skills).

*klgarber@live.unc.edu

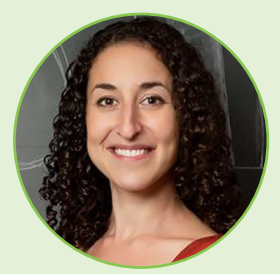

\section{EVA H. TELZER}

I am an Assistant Professor of Psychology and Neuroscience at The University of North Carolina at Chapel Hill. My research uses multimethod tools including longitudinal assessments, $\mathrm{fMRI}$, daily diaries, and diurnal cortisol to examine how social and cultural processes shape brain development. My research focuses on both antisocial (e.g., risk taking and substance use) and prosocial (e.g., helping and empathy) behaviors from childhood to adulthood, with a focus on adolescence as a particularly sensitive and flexible phase of brain development. 